\title{
Influencia del apoyo familiar en la autoeficacia de los drogodependientes.
}

\author{
Francisca López-Torrecillas'; Mercedes Bulas²; Raquel León-Arroyo²; Isabel Ramírez'. \\ ${ }^{1}$ Profesora Asociada. Departamento de Personalidad, Evaluación y Tratamiento Psicológico. Facultad de Psicología. Universidad de Granada. \\ 2 Alumna de Doctorado del Departamento de Personalidad, Evaluación y Tratamiento Psicológico. Facultad de Psicología. Universidad de Granada.
}

Enviar correspondencia a:

Francisca López-Torrecillas. Departamento de Personalidad, Evaluación y Tratamiento Psicológico. Facultad de Psicología. Universidad de Granada. Campus Universitario de Cartuja s/n. 18071- GRANADA. TLF: 958-249557 - FAX: 958-243749. e-mail: fcalopez@platon.ugr.es

Recibido: 8 de Septiembre de 2003. Aceptado: 20 de Julio de 2005

\section{RESUMEN}

En este estudio se investiga si el apoyo de los progenitores afecta a la autoeficacia de los drogodependientes. Para ello se utilizó una muestra de 186 sujetos de Méjico que se dividieron en dos grupos (experimental y control). A su vez, ambos grupos se dividieron en 2 subgrupos en función del apoyo de sus progenitores. Se midió la autoeficacia con la escala de Sherer et al., (1982) y el consumo de drogas con la entrevista de investigación acerca del comportamiento adictivo. Se realizó un Análisis Multivariado de la Varianza (MANOVA) para un diseño bifactorial entre grupos utilizando como variables independientes el consumo (consumo versus no consumo) y el apoyo de los progenitores (implicación versus no implicación) y como variable dependiente la autoeficacia. Los resultados indicaron que los sujetos drogodependientes presentaban puntuaciones más bajas en autoeficacia que los sujetos controles. No se encontraron diferencias estadísticamente significativas en el apoyo de los progenitores aunque podemos observar que el nivel de autoeficacia de los grupos (experimental y control) es menor cuando los progenitores se implican. Nuestros resultados ponen de manifiesto que la autoeficacia podría ser una variable protectora para el consumo de drogas y sugieren la necesidad de seguir investigando las actitudes, actuaciones y recursos familiares que inciden en el consumo de drogas.

Palabras clave: autoeficacia, apoyo familiar, drogodependientes, factores de protección.

\begin{abstract}
This study investigates whether or not parental support affects the self-efficacy of the substance abuser. A sample of 186 subjects from Mexico was divided into two groups (experimental and control). Both groups, in their turn, were divided into 2 subgroups in relation to parental support. Selfefficacy was measured with the scale of Sherer et al., (1982), and drug use with the research interview on addiction behaviour. A Multivariable Analysis of Variance (MANOVA) was carried out for a bifactorial design among groups with use (use versus nonuse) and parental support (involvement versus non-involvement) as independent variables and with self-efficacy as dependent variable. The results indicated that the drug dependent subjects presented lower scorings in self-efficacy than the control subjects. Statistically significant differences in parental support were not found although we do observe that the level of self-efficacy in the groups (experimental and control) is lower when parents are involved. Our results revealed that selfefficacy could be a protective variable in drug use, and suggest the need to continue investigating the familial attitudes, actions and resources that have an impact on drug use.
\end{abstract}

Keywords: self-efficacy, familial support, drug use, protective factor.

características que están presentes en las relaciones entre sus miembros produciendo una influencia constante durante todo el proceso de las drogodependencias (inicio del consumo, mantenimiento del consumo y demanda de tratamiento). Casas y Gossop (1993) afirman que la presión familiar influye en la decisión de abandonar el consumo de alcohol y/o drogas.

El papel de los progenitores sobre su influencia en el proceso de tratamiento de las drogodependencias 
también se ha constatado en algunos estudios (Kosten, Jalali, Hogan y Kleber, 1983; Levy, 1972; Mansilla, Vega, Gómez-Ravecedo y Márquez, 1997). Se observa que la ausencia de ambos padres o de uno de ellos está asociada con un peor pronóstico. Concretamente, Levy (1972), en su estudio de seguimiento de cinco años sobre adictos a narcóticos, encuentra que los sujetos que superaban el hábito contaban habitualmente con respaldo familiar. Kosten et al., (1983) señalan que los heroinómanos que viven con su familia obtienen mejores resultados en el tratamiento que los heroinómanos que viven solos y Mansilla et al., (1997), en su estudio con drogodependientes sin hogar, concluyen que tanto la abstinencia como la retención al tratamiento es difícil en esta población, dado que, uno de los factores que influyen en este fracaso es la disfuncionalidad familiar y/o el escaso o nulo apoyo familiar.

En una línea opuesta, existen una serie de estudios (Vaillant, 1966; Zahn y Ball, 1972) que encuentran que los drogodependientes que superaban con éxito su tratamiento no vivían con sus padres. Por tanto, se asoció la rehabilitación con el no vivir con los padres. En este sentido algunos estudios (Ress y Wilborn, 1983; Stoker y Swadi,1990) asocian las relaciones familiares disfuncionales con el mantenimiento del consumo de alcohol y drogas. Específicamente, Ress y Wilborn (1983) sostienen que los sujetos con trastornos adictivos perciben a sus padres como más rechazantes, irritables, negligentes, intrusivos, posesivos, sobreprotectores, manipuladores de sentimientos de culpa y sin una dirección y criterio precisos en las reglas. Por su parte, Stoker y Swadi (1990) encuentran que los consumidores de alcohol y drogas describen la relación entre sus padres como distante y aislada, con frecuentes discusiones y conflictos, con dificultades de comunicación entre ambos padres, quiénes, a su vez, tienden a ser desconfiados, verbalmente punitivos y críticos respecto al hijo. Además, las madres son percibidas por sus hijos drogodependientes como más punitivas, con relaciones frecuentes, pero más negativas. Los padres son considerados por sus hijos drogodependientes como más ineficaces y ausentes. Johnson y Pandina (1991a,b) encuentran que los sujetos consumidores de alcohol y/o drogas describían las relaciones parentales como aversivas frente a los no consumidores que las describían como afectivas. La percepción de los hijos drogodependientes acerca de sus padres provocan estados de ánimo negativos que facilitan el mantenimiento del comportamiento adictivo (Ellis y Mclnerny, 1992).

Otros resultados también nos sugieren que los padres que no disponen de habilidades para resolver dificultades de la vida diaria tienen hijos que consumen alcohol y drogas. Garvey (2000) afirma que las actitudes, los estilos parentales, la armonía y cohe- sión afecta al afrontamiento del consumo de alcohol y drogas de sus hijos.

Parece, por tanto, que la ausencia de apoyo familiar, la disfuncionalidad en las relaciones y la falta de habilidades para resolver problemas con los hijos están relacionadas con el consumo de alcohol y/o drogas. De acuerdo con (Booth, Russell, Soucek y Laughlin, 1992; Schneider, 1977; Staton, 1978), un drogodependiente puede tener una relación familiar orientada hacia dos extremos: la sobreimplicación de la madre y la baja implicación del padre, pero ninguno de los dos casos necesariamente conlleva afecto y aceptación paterna. Por lo tanto, parece ser que la implicación familiar no conlleva necesariamente afecto. Sería entonces la percepción del drogodependiente de la ausencia de aceptación y amor por parte de los padres, más que los extremos de implicación, el factor que contribuye al inicio y al mantenimiento del abuso de drogas

No podemos negar que los progenitores ejercen una notable influencia sobre el inicio y mantenimiento de la conducta adictiva de sus hijos. Sus actitudes y valores, los mensajes implícitos y explícitos, la manera de relacionarse, de solucionar conflictos o gestionar emociones ejercen un notable impacto sobre el ajuste psicosocial de sus hijos. Las pautas de comportamiento hacia sus hijos pueden facilitar o dificultar el ajuste conductual, emocional y social de estos. Es necesario tener en cuenta que la calidad de la presión, el soporte familiar y los recursos sociales disponibles están involucrados en el fenómeno de las drogodependencias.

En esta línea, otros estudios (Bishop, 1995; Hoelter y Harper, 1987; Jacob y Seilhamer, 1985) ponen de manifiesto que en las drogodependencias no es crucial la presencia o ausencia de los padres sino la naturaleza de la relación familiar. Las habilidades de manejo, definidas en función de la competencia versus incompetencia para establecer normas de comportamiento y recompensar o castigar adecuadamente, son interiorizadas por los hijos y afectan al desarrollo del autoconcepto y autoestima. Concretamente, Booth et al., (1992) encuentran que el apoyo familiar que recibían los drogodependientes en tratamiento producía un aumento de su autoestima y autoeficacia y consecuentemente no abandonaban el tratamiento, en comparación, con el apoyo familiar que recibían otros drogodependientes que no producía ningún aumento de su autoestima y autoeficacia y consecuentemente abandonaban el tratamiento.

Aunque no disponemos de más estudios que nos avalen que los padres desarrollan estrategias y comportamientos de relación con sus hijos que orientan a estos hacia un ajuste (promover sus competencias personales) versus desajuste personal (promover acciones que faciliten el consumo de alcohol y/o dro- 
gas), cabe plantearse la influencia de los padres en el estilo de vida del hijo y en sus competencias personales (autoeficacia). Se hace necesario, por tanto, seguir indagando sobre esta relación. No nos referimos al apoyo versus no apoyo familiar sino a como afecta el apoyo familiar a las competencias personales del drogodependiente.

El papel de la autoeficacia en el consumo de alcohol y/o drogas ha sido ampliamente estudiado y constatado (Baldwin, Oei y Young 1993; Bell, Ellickson y Harrison, 1993; Hays y Ellickson, 1990; Ellickson y Hays, 1991, 1992; Pentz, 1985; Sadowski, Long y Jenkins, 1993; Sitharthan y Kavanagh,1990). La autoeficacia predice el consumo de alcohol y/o drogas, esto es, se relaciona una baja autoeficacia con una frecuencia más alta y una mayor cantidad de consumo de alcohol y/o drogas.

\section{OBJETIVOS.}

En consonancia con lo anterior el objetivo de este estudio es analizar la influencia del apoyo familiar en la autoeficacia de los drogodependientes.

\subsection{Hipótesis.}

De este objetivo se derivan dos hipótesis:

a. Los sujetos que consumen alcohol y drogas presentarán puntuaciones más bajas en autoeficacia que los no consumidores.

b. Los sujetos que consumen alcohol y drogas y disponen de implicación parental presentarán puntuaciones más altas en autoeficacia que aquellos que no disponen de implicación familiar y también consumen alcohol y drogas.

\section{3.- METOdOLOGÍA.}

\subsection{Sujetos.}

Participaron en este estudio 186 sujetos, 149 hombres y 37 mujeres, con una edad media de 17.79 años y desviación típica de 2.74, procedentes de la Puebla (Méjico). Los sujetos fueron divididos en 2 grupos (grupo experimental y grupo control). El grupo experimental fue seleccionado en Centros de Integración Juvenil, instituciones especializadas en el tratamiento del consumo de drogas. Los sujetos de este grupo acudían a solicitar tratamiento. La selección del grupo de control se hizo en la institución educativa CONALEP, los sujetos de este grupo acudían a la institución para realizar estudios o asesoramiento laboral. Los grupos (experimental y control) fueron divididos en función del apoyo que recibían por parte de sus progenitores. Por tanto, los grupos quedaron formados de la siguiente manera:

Grupo Experimental: Consumidores de alcohol y drogas $(n=109)$ compuesto por 91 hombres y 18 mujeres, con una edad media de 18.54 años y desviación típica de 2.91. Los sujetos que componen este grupo acudían al centro para recibir tratamiento de su drogodependencia y presentaban diferente grado de implicación de los padres (implicación versus no implicación).

- Grupo 1 (experimental): Consumidores de alcohol y drogas cuyos padres (padre y/o madre o ambos) se implican $(n=67)$ compuesto por 58 hombres y 9 mujeres, con una edad media de 18.12 años y desviación típica de 2.82. Los sujetos que componen este grupo acudían al centro para recibir tratamiento de su drogodependencia e iban acompañados de sus progenitores (padre y/o madre o ambos).

- Grupo 2 (experimental): Consumidores de alcohol y drogas cuyos padres no se implican $(n=42)$ compuesto por 33 hombres y 9 mujeres, con una edad media de 19.21 años y desviación típica de 2.96 .

Grupo Control: No consumidores de alcohol y drogas $(n=77)$ compuesto por 53 hombres y 15 mujeres, con una edad media de 16.74 años y desviación típica de 2.10. Los sujetos de este grupo acudían al centro para estudiar y/o recibir asesoramiento laboral y presentaban diferente grado de implicación de los padres (implicación versus no implicación).

- Grupo 3 (control): No consumidores de alcohol y drogas cuyos progenitores (padre y/o madre o ambos) se implican $(n=51)$ compuesto por 38 hombres y 13 mujeres con una edad media de 16.53 años y desviación típica de 1.92. Los sujetos de este grupo acudían al centro para estudiar y/o recibir asesoramiento laboral. Cuando se requería la participación de los padres siempre acudían los progenitores (padre y/o madre o ambos) En el momento de la evaluación iban acompañados de sus progenitores (padre y/o madre o ambos).

- Grupo 4 (control): No consumidores de alcohol y drogas cuyos padres no se implican $(n=26)$ compuesto por 6 mujeres y 20 hombres con una edad media de 17.15 años y desviación típica de 2.39. Los sujetos de este grupo acudían al centro para estudiar y/o recibir asesoramiento laboral. Cuando se requería la participación de los padres 
nunca acudían. En el momento de la evaluación iban solos.

En la adscripción de los sujetos a los grupos se controló la edad $\left(F_{1,182}=0.343 ;\right.$ Mce $\left.=2.286 ; p=0.559\right)$, nivel educativo $\left(F_{1,182}=0.200, M c e=1.015, p=0.655\right)$, género $\left(\chi^{2}(1)=1.886\right.$; $\left.p=0.170\right)$ y severidad del consumo de drogas. Para controlar esta última variable realizamos, al igual que con la variable edad y nivel educativo, un Análisis de Varianza (ANOVA), para un diseño bifactorial entregrupos $2 \times 2$, utilizando como variables independientes el consumo (consumo versus no consumo) y el grado de implicación parental (implicación versus no implicación) y como variable dependiente la puntuación estandarizada de la severidad en el consumo de alcohol y drogas (cantidad y cronicidad del consumo de alcohol, hachís, anfetaminas, metadona, alucinógenos, cocaína, heroína y medicamentos). Los resultados indicaron diferencias significativas en el factor grupo $\left(F_{1,182}=86.29\right.$, Mce=3174.408, $\mathrm{p}=0.000$ ). No se encontraron diferencias estadísticamente significativas para la interacción $\left(F_{1,182}=0.010\right.$, Mce $=0.373, p=0.920)$ y para el factor grado de implicación $\left(F_{1,182}=0.19\right.$, Mce $\left.=0.682, p=0.892\right)$. Por lo que se demuestra que la severidad del consumo de alcohol y/o drogas no afecta a nuestras variables de estudio. En la tabla 1 podemos observar las características sociodemográficas (edad, nivel educativo y género) de los sujetos que componen la muestra del estudio em función de los diferentes grupos y en la tabla 2 podemos observar las medias y desviaciones típicas de la severidad del consumo de alcohol y drogas de los grupos experimental y control, con los subgrupos de implicación (implicación versus no implicación).

TABLA 1: Medias y desviaciones típicas de la edad, nivel educativo y número de sujetos en función del género de los grupos experimentales y controles en sus diferentes niveles de implicación familiar.

\begin{tabular}{|c|c|c|c|c|}
\hline VARIABLES & GRUPOS & $\begin{array}{l}\text { GRADO DE } \\
\text { IMPLICACIÓN }\end{array}$ & MEDIA & $\begin{array}{l}\text { DESVIACIÓN } \\
\text { TíPICA }\end{array}$ \\
\hline \multirow[t]{2}{*}{ EDAD } & EXPERIMENTAL & $\begin{array}{l}\text { IMPLICADOS } \\
\text { NO IMPLICADOS }\end{array}$ & $\begin{array}{l}18.12 \\
19.21\end{array}$ & $\begin{array}{l}2.82 \\
2.95\end{array}$ \\
\hline & CONTROL & $\begin{array}{l}\text { IMPLICADOS } \\
\text { NO IMPLICADOS }\end{array}$ & $\begin{array}{l}16.53 \\
17.15\end{array}$ & $\begin{array}{l}1.92 \\
2.39\end{array}$ \\
\hline \multirow[t]{2}{*}{ NIVEL EDICATIVO } & EXPERIMENTAL & $\begin{array}{l}\text { IMPLICADOS } \\
\text { NO IMPLICADOS }\end{array}$ & $\begin{array}{l}9.36 \\
9.90\end{array}$ & $\begin{array}{l}2.38 \\
2.47\end{array}$ \\
\hline & CONTROL & $\begin{array}{l}\text { IMPLICADOS } \\
\text { NO IMPLICADOS }\end{array}$ & $\begin{array}{l}9.88 \\
10.12\end{array}$ & $\begin{array}{l}2.29 \\
1.24\end{array}$ \\
\hline & & & HOMBRES & MUJERES \\
\hline \multirow[t]{2}{*}{ GÉNERO } & EXPERIMENTAL & $\begin{array}{l}\text { IMPLICADOS } \\
\text { NO IMPLICADOS }\end{array}$ & $\begin{array}{l}58 \\
32\end{array}$ & $\begin{array}{l}9 \\
9\end{array}$ \\
\hline & CONTROL & $\begin{array}{l}\text { IMPLICADOS } \\
\text { NO IMPLICADOS }\end{array}$ & $\begin{array}{l}38 \\
20\end{array}$ & $\begin{array}{l}13 \\
6\end{array}$ \\
\hline
\end{tabular}

TABLA 2: Medias y desviaciones típicas de la puntuación total estandarizada del consumo de alcohol y drogas de los grupos en sus diferentes niveles de implicación familiar.

\begin{tabular}{|l|l|l|c|c|}
\hline VARIABLES & GRUPOS & $\begin{array}{l}\text { GRADO DE } \\
\text { IMPLICACIÓN }\end{array}$ & MEDIA & $\begin{array}{c}\text { DESVIACIÓN } \\
\text { TíPICA }\end{array}$ \\
\hline $\begin{array}{l}\text { SEVERIDAD DEL } \\
\text { CONSUMO DE }\end{array}$ & EXPERIMENTAL & IMPLICADOS & 3.47 & 9.05 \\
ALCOHOL Y & NO IMPLICADOS & 3.70 & 5.56 \\
\cline { 2 - 5 } DROGAS & \multirow{2}{*}{ CONTROL } & IMPLICADOS & -5.20 & 0.52 \\
& & NO IMPLICADOS & -5.16 & 0.64 \\
\hline
\end{tabular}




\subsection{Medidas.}

\subsubsection{Escala de Autoeficacia de Sherer, Maddux, Mercandante, Prentice-Dunn, Jacobs y Rogers (1982) (A.E.).}

Esta escala fue desarrollada para analizar las creencias que tienen los sujetos acerca de sus habilidades para manejar determinadas situaciones. Traducida por Godoy (1990).

Consta de 23 ítems, divididos en 2 subescalas. En la subescala de Autoeficacia General, el sujeto describe cómo maneja determinadas situaciones relacionadas con tareas personales. En la subescala de Autoeficacia Social, el sujeto describe cómo maneja determinadas situaciones sociales. En ambas escalas el sujeto debe indicar el grado de acuerdo o desacuerdo con la frase que le describe o caracteriza en una escala de 1 a 5 , donde 1 significa estar fuertemente en desacuerdo y 5 fuertemente de acuerdo.

Con respecto a la fiabilidad, Sherer et al., (1982) señalan una consistencia interna, con el coeficiente alpha de Cronbach de 0.86 para la subescala de Autoeficacia General y de 0.71 para la subescala de Autoeficacia Social. Por su parte, Woodruff y Cashman (1993) obtienen un coeficiente alpha de Cronbach de 0.84 para la subescala de Autoeficacia General y de 0.69 para la subescala de Autoeficacia Social.

Más recientemente Washington (1999) encuentra una fiabilidad con el procedimiento test-retest, de 0.77 , y con el método de las dos mitades, de 0.77. Mattoo y Malhotra (1998) obtienen una consistencia interna, con el coeficiente alpha de 0.74 para la subescala de Autoeficacia General y de 0.99 para la subescala de Autoeficacia Social y 1 para la escala total. Con el procedimiento test-retest, de 0.91 para la subescala de Autoeficacia General y de 0.94 para la subescala de Autoeficacia Social y para la escala total de 0.93 .

Con respecto a la aportación de otras evidencias de validez, Sherer y Adams (1983); Sherer et al. (1982) señalan una adecuada validez convergente con otras medidas de características de personalidad, relacionadas con la eficacia general sin ser sinónimas.

En lo que respecta a las evidencias de validez predictiva encontramos, que la escala predice comportamientos dirigidos hacia el control de consumo de alcohol (Baldwin et al, 1993), el mantenimiento de la abstinencia de los drogodependientes (Sadowski et al., 1993) y .la recaída en los sujetos drogodependientes después de haber realizado tratamiento para su dependencia (López- Torrecillas, de la Fuente, Castillo, Galindo y Ramírez, 2000)

Con respecto a las evidencias de validez discriminante, los estudios muestran que la escala es un instrumento muy eficaz para identificar poblaciones de riesgo (Godoy et al., 1995; Laserna et al., 1997).

\subsubsection{Entrevista de Investigación acerca del Com- portamiento Adictivo (E.I.C.A).}

Detecta el consumo de alcohol y drogas en el momento de la evaluación, versa sobre el comportamiento adictivo, particularmente del consumo de alcohol, hachís, anfetaminas, metadona, alucinógenos, cocaína, heroína y medicamentos. Hace referencia a la frecuencia de consumo de estas sustancias, cantidad de consumo y edad de inicio de las mismas. Consta de 8 ítems referidos a cada una de las sustancias que, a su vez, se dividen en tres grupos de preguntas. El primer grupo hace referencia a la frecuencia de consumo en el último mes que puede ser a diario, entre una y tres veces a la semana, una vez a la semana, entre una y tres veces al mes y una vez al mes. El segundo grupo hace referencia a los gramos, mililitros o unidades y el tercer grupo hace referencia al tiempo de consumo en años desde que se inició este. Con este último grupo calculamos la cronicidad del consumo de drogas que se puntúa en años y se valora el tiempo de consumo desde la edad de inicio del consumo hasta el momento de la evaluación. Con el primer y segundo grupo de preguntas calculamos la cantidad de drogas consumidas en el último mes, el primer grupo es indicativo de sí el consumo es diario, semanal o mensual, se puntúa desde 1 hasta 8 y oscila desde nunca hasta a diario; el segundo grupo es indicativo del número de copas, cigarrillos o gramos que se consumen, se puntúa teniendo en cuenta que un whisky equivale a 1 unidad y un vino o una cerveza equivale a 0.5 , el hachís se puntúa en número de cigarrillos y la cocaína y heroína en gramos al mes. En el paso siguiente se obtienen 16 medidas que informan sobre la frecuencia de uso y unidades de consumo o cantidad de consumo de drogas (cantidad de consumo de alcohol, hachís, anfetaminas, metadona, alucinógenos, cocaína, heroína y medicamentos), así como los años transcurridos desde que se inició el consumo o cronicidad de consumo de drogas (cronicidad de consumo de alcohol, hachís, anfetaminas, metadona, alucinógenos, cocaína, heroína y medicamentos). Finalmente se obtienen 2 puntuaciones estandarizadas que definen el consumo de drogas, una es la cantidad de consumo de drogas, en su puntuación típica y otra es la cronicidad de dicho consumo, en su puntuación típica.

\subsection{Variables y análisis estadísticos.}

\subsubsection{Variables Dependientes:}

- Puntuación de autoeficacia derivada de la Escala de Autoeficacia de Sherer et al. (1982) en sus tres dimensiones:

- Autoeficacia general (AEG).

- Autoeficacia social (AES).

- Autoeficacia total (AET).

3.3.2. Variables Independientes: 
- Consumo medido a través de la entrevista de investigación acera del comportamiento adictivo y manipulado en dos niveles:

- Grupo de consumo o experimental.

- Grupo de no consumo o control.

- Apoyo de los progenitores medido a través de la información recogida de los profesionales de los centros (Centro de Integración Juvenil y Centro Educativo CONALEP) y contrastada en el momento de la evaluación, manipulado en dos niveles:

- Padres implicados (padre, madre o ambos).

- Ninguna implicación por parte de los padres.

Para demostrar las hipótesis se realizó un Análisis Multivariado de la Varianza (MANOVA) para un diseño bifactorial entregrupos $2 \times 2$, utilizando como variables independientes el consumo (consumo versus no consumo) y el grado de implicación parental (implicación versus no implicación) y las variables de autoeficacia (autoeficacia general, autoeficacia social y autoeficacia total) como variables dependientes.

\subsection{Procedimiento.}

En primer lugar se procedió a la toma de contacto con la muestra mediante una entrevista inicial individualizada, en ella se proporcionaba información sobre la investigación y se preguntaba al sujeto si le interesaría participar en ella. En caso de obtener una respuesta afirmativa, se procedía a obtener información de los profesionales de los centros (Centro de Integración Juvenil y Centro Educativo CONALEP). Se les pedía que nos indicaran quien era el miembro de la familia con actitud de disponibilidad cuando se requería cualquier información o apoyo de la familia tanto en el tratamiento de las drogodependencias, en el caso del grupo experimental, como en el asesoramiento laboral o escolar, en el caso del grupo de control. En segundo lugar, se procedía a la recogida de los datos sociodemográficos y a cumplimentar de la Entrevista de Información acerca del Comportamiento Adictivo y de la Escala de Autoeficacia. La evaluación era individualizada y las normas de aplicación eran las estandarizadas en cada prueba. En este momento, también se preguntaba al sujeto quien era el miembro de la familia que adoptaba una actitud de disponibilidad cuando los profesionales del centro requerían a la familia para apoyo de actividades de tratamiento, educativas o de asesoramiento laboral.

\section{RESULTADOS.}

Para demostrar las hipótesis que planteamos en este estudio, se procedió a comprobar si la variable autoeficacia diferenciaba a los grupos (experimental y control) en función del grado de implicación familiar (implicación versus no implicación). Para ello, se realizó un Análisis Multivariado de la Varianza (MANOVA), para un diseño bifactorial entregrupos $2 \times 2$ utilizando como variables independientes el consumo (consumo versus no consumo) y el grado de implicación parental y las variables de autoeficacia (autoeficacia general, autoeficacia social y autoeficacia total) como variables dependientes. Los resultados mostraron diferencias estadísticamente significativas en el factor consumo (Wilks' Lambda $=0.865, F_{3,180}=9.38 ; p<$ .001) con un porcentaje de explicación del 14\%. No se encontraron diferencias estadísticamente significativas para el factor grado de implicación (Wilks' Lamb$\left.\mathrm{da}=0.995, F_{3,180}=0.313, p<.01\right)$ y para la interacción (Wilks' Lambda $=0.997, F_{3,180}=0.201, p=.896$ ).

Puesto que el MANOVA mostró resultados estadísticamente significativos en el factor consumo, se procedió a realizar ANOVAs bifactoriales univariados para cada uno de los niveles de la variable dependiente (autoeficacia general, autoeficacia social y autoeficacia total) utilizando como variables independientes el consumo y el grado de implicación. Dichos ANOVAs mostraron que había diferencias estadísticamente significativas en la autoeficacia general $\left(F_{1,182}\right.$ $=10.84$; Mce $=822.10 ; p<.001)$ con un porcentaje de explicación del $5.6 \%$, en la autoeficacia social $\left(F_{1,182}\right.$ $=12.43$; Mce $=222.689 ; p<.001$ ) con un porcentaje de explicación del $6.5 \%$ y en la autoeficacia total $\left(F_{1,182}=20.17 ;\right.$ Mce $\left.=2278.707 ; p<.001\right)$ con un porcentaje de explicación del $10 \%$ siendo, en los tres casos, las puntuaciones más bajas para el grupo experimental que para el grupo control. En la tabla 3 se puede observar las medias, desviaciones típicas de las puntuaciones de autoeficacia general, autoeficacia social y puntuación total de autoeficacia así como la significatividad y los etas cuadrados.

\section{DISCUSIÓN Y CONCLUSIONES.}

Los resultados señalan diferencias estadísticamente significativas en el factor consumo con respecto a la autoeficacia general, social y a la puntuación total siendo, en los tres casos, las puntuaciones más bajas para el grupo experimental que para el grupo control, esto es, los sujetos que consumen alcohol y drogas presentan puntuaciones más bajas en autoeficacia que los no consumidores. Estos resultados van en concordancia con los obtenidos Ellickson y Hays (1991) en el que se encontró diferencias en autoeficacia entre sujetos consumidores y no consumidores de drogas, siendo igualmente para los no consumidores la puntuación en dicha variable más alta; con los resultados obtenidos por Bell et al. (1993) quienes hallaron que los sujetos que inician su consumo de alcohol y drogas presentan 
TABLA 3: Medias, desviaciones típicas y significatividad de la variable autoeficacia de los grupos experimentales y controles en sus diferentes niveles de implicación familiar.

\begin{tabular}{|c|c|c|c|c|c|c|}
\hline VARIABLES & GRUPOS & $\begin{array}{l}\text { GRADO DE } \\
\text { IMPLICACIÓN }\end{array}$ & MEDIA & $\begin{array}{l}\text { DESV. } \\
\text { TíPICA }\end{array}$ & $F$ & $\begin{array}{c}\text { Eta } \\
\text { cuadrado }\end{array}$ \\
\hline \multirow[t]{2}{*}{$\begin{array}{l}\text { AUTOEFICACIA } \\
\text { GENERAL }\end{array}$} & EXPERIMENTAL & $\begin{array}{l}\text { IMPLICADOS } \\
\text { NO IMPLICADOS }\end{array}$ & $\begin{array}{l}56.13 \\
57.74\end{array}$ & $\begin{array}{c}7.32 \\
10.03 \\
\end{array}$ & \multirow{2}{*}{$10.84^{* * *}$} & \multirow{2}{*}{0.056} \\
\hline & CONTROL & $\begin{array}{l}\text { IMPLICADOS } \\
\text { NO IMPLICADOS }\end{array}$ & $\begin{array}{l}61.25 \\
61.53\end{array}$ & $\begin{array}{c}7.26 \\
11.84\end{array}$ & & \\
\hline \multirow[t]{2}{*}{$\begin{array}{l}\text { AUTOEFICACIA } \\
\text { SOCIAL }\end{array}$} & EXPERIMENTAL & $\begin{array}{l}\text { IMPLICADOS } \\
\text { NO IMPLICADOS }\end{array}$ & $\begin{array}{l}19.48 \\
18.90\end{array}$ & $\begin{array}{c}7.70 \\
10.83\end{array}$ & \multirow{2}{*}{$12.43^{* * *}$} & \multirow{2}{*}{0.064} \\
\hline & CONTROL & $\begin{array}{l}\text { IMPLICADOS } \\
\text { NO IMPLICADOS }\end{array}$ & $\begin{array}{l}21.55 \\
21.54\end{array}$ & $\begin{array}{l}5.07 \\
3.88\end{array}$ & & \\
\hline \multirow[t]{2}{*}{$\begin{array}{l}\text { PUNTUACIÓN } \\
\text { TOTAL }\end{array}$} & EXPERIMENTAL & $\begin{array}{l}\text { IMPLICADOS } \\
\text { NO IMPLICADOS }\end{array}$ & $\begin{array}{l}74.46 \\
76.64\end{array}$ & $\begin{array}{l}3.5 \\
4.11\end{array}$ & \multirow{2}{*}{$20.17^{* * *}$} & \multirow{2}{*}{0.1} \\
\hline & CONTROL & $\begin{array}{l}\text { IMPLICADOS } \\
\text { NO IMPLICADOS }\end{array}$ & $\begin{array}{l}82.80 \\
83.15\end{array}$ & $\begin{array}{l}8.57 \\
13.60\end{array}$ & & \\
\hline
\end{tabular}

puntuaciones más bajas en autoeficacia que aquellos que no consumen, dado que, los sujetos que inician el consumo se sienten más incapaces para rehusar al consumo de drogas que los sujetos que no consumen; también son consistentes con los resultados encontrados por Baldwin et al. (1993) en donde las puntuaciones bajas en autoeficacia están relacionadas con el consumo de alcohol en una muestra de estudiantes y finalmente, con los resultados obtenidos por Sadowski et al.(1993) quienes obtuvieron, mediante la implantación de un programa de entrenamiento en Autoeficacia a 142 sujetos consumidores, que puntuaciones altas en Autoeficacia mantenían la abstinencia de estos sujetos. En definitiva esto se traduce a que los sujetos que puntúan bajo en autoeficacia tienden a consumir alcohol y/o drogas y los que puntúan alto en autoeficacia no consumen.

Si bien no encontramos diferencias estadísticamente significativas para el factor implicación de los padres, podríamos hablar de diferencias clínicas que pasamos a analizar: En primer lugar, para el caso del grupo experimental, observamos que los sujetos que disponen de implicación de los padres puntúan más bajo en autoeficacia general y en la puntuación total que los sujetos que no disponen de implicación de los padres. En segundo lugar, se observa también, que las tendencias anteriormente mencionadas no se obtienen para el caso de la autoeficacia social, es decir, los sujetos consumidores de alcohol y drogas que disponen de implicación parental puntúan más alto en autoeficacia social que aquellos que no disponen de implicación parental y también consumen alcohol y drogas. Por otro lado, en tercer lugar, con respecto al grupo control estas diferencias clínicas no aparecen. Si bien no podemos realizar ninguna conclusión parece que estos resultados van en la línea de los obtenidos por Bishop (1995); Hoelter y Harper (1987); Jacob y Seilhamer (1985) quienes resaltan la importancia de la naturaleza de la relación familiar en las drogodependencias.

Teniendo en cuenta todo esto, los programas de prevención de consumo de alcohol y/o drogas, así como los programas de tratamiento deberían incluir como una de las piezas clave de su intervención la autoeficacia, para incrementar y/o posibilitar el desarrollo de habilidades de afrontamiento ante situaciones que inciten al consumo así como estrategias de evitación o control de dicho consumo.

Por otro lado, resaltamos la necesidad de seguir investigando el papel de la familia en el campo de las drogodependencias, dado que, sigue estando poco claro. Actualmente esta siendo contemplada la influencia familiar en los trastornos adictivos y sabemos que estilos educativos inadecuados se asocian con el inicio y mantenimiento del consumo de alcohol y drogas, que los conflictos familiares afectan al estado emocional de sus miembros y consecuentemente pueden alterar el proceso de rehabilitación del drogodependiente, que los padres sobreprotectores se consideran más ineficaces para manejar las conductas adictivas de sus hijos y que la familia del drogodependiente presenta déficit en la comunicación y alineación. Seguimos sin saber con exactitud las acti- 
tudes, actuaciones y recursos que influyen en el inicio del consumo de alcohol y/o drogas, en la decisión de demandar tratamiento y en las recaídas.

\section{REFERENCIAS.}

Baldwin, A.R., Oei, T.P.S. y Young, R. (1993). To drink or not to drink: The differential role of alcohol expectancies and drinking refusal self-efficacy in quartely and frequency of alcohol consumption. Cognitive Therapy and Research, 17, 511-530.

Bell, R.M., Ellickson, P.L. y Harrinson, E.R. (1993). Do drug prevention effects persist into high school?. How project alert did with granders. Preventive Medicine, 22, 463-483.

Bishop, J.A. (1995). Peer acceptance and friendship: An investigation of their relation to self-esteem. Journal of Early Adolescence, 15, 476-489.

Booth, B.M.; Russell, D.W.; Soucek, S. y Laughlin, P.R. (1992). Social support and outcome of alcoholism treatment: an exploratory analysis. American Journal Drug Alcohol Abuse, 18, 87-101.

Casas, M. y Gossop, M. (1993). Tratamientos psicológicos en drogodependencias. Recaída y prevención de recaídas. Barcelona: Generalitat de Catalunya.

Ellickson, P. L y Hays, R.D. (1991). Antecedents of drinking among young adolecescents with different alcohol use histories. Journal of Studies on Alcohol, 52, 398-408.

Ellis, A. y Mclnerny, J.F. (1992). Terapia racional-emotiva con alcohólicos y toxicómanos. Bilbao: DBD.

Garvey, K.A. (2000). Family members and significant other of drug abusers: Characteristics of the relationship with the drug abuser and predictors of outcome in unilateral treatment. Dissertation Abstracts International, 60, 8.

Godoy, J.F. (1990). Escala de autoeficacia. Versión experimental, no publicada.

Godoy, J.F., Muela, J.A., Sánchez-Barrera, M.B., SánchezHuete, J.R., Pérez, M., Martínez, J.M., Anguiano, I., Lorite, A. y Marí-Beffa, P. (1995). Marcadores de vulnerabilidad/factores de protección en la prevención de la esquizofrenia. Psicología Conductual, 3, 35-46.

Hays, R.D. y Ellickson, P.L. (1990). How generalizable are adolescents' beliefs about pro-drug pressures and resistance self-efficacy?. Journal of Applied Social Psychology, 20, 321-340.

Hoelter, J. y Harper, L. (1987). Structural and interpersonal family influences on adolescent self-conception. Journal of Marriage and the Family, 49, 129-139.

Jacob, T. y Seilhamer, R.A. (1985). Adaptation of the areas of change questionnaire for parent-child relationship assessment. American Journal of Family Therapy, 13, 28-38.
Johnson, V. y Pandina, R.J. (1991a). Familial and personal drinking histories and measures of competence in youth. Addictive Behaviors, 16, 453-465.

Johnson, V., y Pandina, R.J. (1991b). Effects of the family environment on adolescent substance use, delinquency and doping styles. American Journal of Drug Alcohol and Abuse, 17, 71-88.

Kosten, T.R.; Jalali, B.; Hogan, I.K. y Kleber, H.D. (1983). Family denial as a prognostic factor in opiate addict treatment outcome. Journal of Nervous and Mental Disease, 171, 611-616.

Laserna, J.A., Castillo, A., Peláez, E., Navío, L.F., Torres, C.J., Rueda, S., Ramírez, M.N. y Pérez, M. (1997). Alteraciones emocionales y variables moduladoras en familiares-cuidadores de enfermos de Alzheimer. Psicología Conductual, 5, 365-375.

Levy, B. (1972). Five years after: A follow-up of 50 narcotic addicts. American Journal Psychiatry, 7, 22.

López- Torrecillas, F., de la Fuente, E.I., Castillo, E., Galindo, J. y Ramírez, I. (2000, noviembre). La recaída en las drogodependencias: Efecto modulador de la autoeficacia. Póster presentado en el / Symposium sobre Avances en el Tratamiento Cognitivo-Conductual de los Problemas Psicológicos, Granada.

Mansilla, F.; Vega, P.; Gómez-Ravecedo, M.M. y Márquez, P. (1997). Drogodependientes sin hogar: una experiencia. Revista Española de Drogodependencias, 22, 105113.

Mattoo, S.K. y Malhotra, R. (1998). Self-efficacy Scale: Hindi translation and factor structure. Indian Journal of Clinical Psychology, 25, 154-158.

Pentz, M. (1985). Social competence and self-efficacy as determinants of determinants of substance abuse in adolescence. En: T. A. Wills y S. Shiffman (Eds.), Coping and substance use. (pp. 117-142). New York: Academic Press.

Rees, C.D. y Wilborn, B.L. (1983). Correlates of drug abuse in adolescents: a comparison of families of drug abusers with families of non drug abusers. Journal of Youth and Adolescence, 12, 55-63.

Sadowski, C.J., Long, C.L. y Jenkins, L.R. (1993). Does substance abuse treatment have self-schematic effects?. The Journal of Psychology, 127, 323-327.

Schneider, R.J.; Kojak, G. y Ressdorf, H. (1977). Fatherdistance and drug abuse in young men. Journal of Nervous and Mental Disease, 165, 269-274

Sherer, M., y Adams, C.H. (1983). Construct validation of the Self-efficacy Scale. Psychological Report, 53, 899902.

Sherer, M. Maddux, J.E., Mercandante, B. Prentice-Dunn, S., Jacobs, B., y Rogers, R.W. (1982). The Self-efficacy Scale: Construction and validation. Psychological Reports. 51, 663-671.

Sitharthan, T. y Kavanagh, D.J. (1990). Role of selfefficacy in predicting outcomes from a programme for 
controlled drinking. Drug and Alcohol Dependence, 27, 87-94.

Stanton, M.D (1978). The family and drug misuse: a bibliography. Am J Drug Alcohol Abuse; 5, 151-170.

Stoker, A. y Swadi, H. (1990). Perceived family relationships in drug abusing adolescents. Drug and Alcohol Dependence, 25, 293-297.

Vaillant, E.E. (1966). A 12 year follow-up of New York narcotic addicts III. Some social and Psychiatric characteristics. Archives of General Psychiatry, 15.
Washington, O. (1999). Effects of cognitive and experimental group therapy on self-efcacy and perceptios of employability of chemical dependent women. Issues in Mental Health Nursing, 20, 181198.

Woodruff, S.L. y Cashman, J.F. (1993). Task, domain and general efficacy: A reexaminatio of the Self-efficacy Scale. Psychological Reports,72, 423-432.

Zahn, M. y Ball, J. (1972). Factors related to the cure of opiate addiction among Puerto Rican addicts. $J$ of the Addictions, 7. 
